first steps in environmentally compatible fly control and will provide long-term discouragement of additional pest invaders.

I recommend the following systematic approach: (1) prioritized leadership and hospital administrative commitment to sanitation; (2) empowering and educating food service workers in illness prevention; (3) emphasizing environmentally compatible sanitation methods coupled with strategic and specific applications of antipest agents when required; and (4) looking beyond surface cleanliness to follow food as an attractor of pests.

Acknowledgments. Kurt C. Stange, $\mathrm{MD}, \mathrm{PhD}$, provided helpful guidance in writing this commentary.

Financial support. No financial support was provided relevant to this article.
Conflicts of interest. The author reports no conflicts of interest relevant to this article.

\section{References}

1. Khamesipour F, Lankarani KB, Honarvar B, Kwenti TE. A systematic review of human pathogens carried by the housefly (Musca domestica L.). BMC Public Health 2018;18:1049.

2. Grantz, N. Vector-and Rodent-Borne Diseases in Europe and North America. Cambridge, UK: Cambridge University Press, 2006.

3. Giraldo YM, Leitch KJ, Ros IG, et al. Sun navigation requires compass neurons in Drosophila. Curr Biol 2018;28:2845-2852.

4. Armed Forces Pest Management Board. Filth flies: significance and control in contingency operations. Office of the Under Secretary of Defense website. https://www.acq.osd.mil/eie/afpmb/docs/techguides/tg30.pdf. Published April 2002. Accessed December 28, 2019.

\title{
High minimum inhibitory concentrations among derepressed AmpC-beta-lactamase-producing Enterobacter cloacae complex isolates for ceftolozane with tazobactam
}

\author{
Leandro Reus Rodrigues Perez $\mathrm{PhD}^{1}$ (1), Eliana Carniel BSc ${ }^{2}$ and Gabriel Azambuja Narvaez MD ${ }^{3}$ \\ ${ }^{1}$ Universidade Federal de Ciências da Saúde de Porto Alegre, Porto Alegre, State of Rio Grande do Sul, Brazil, ${ }^{2}$ Universidade Feevale, Novo Hamburgo, State of \\ Rio Grande do Sul, Brazil and ${ }^{3}$ Hospital Mãe de Deus, Porto Alegre, State of Rio Grande do Sul, Brazil
}

To the Editor-Enterobacterales, such as Enterobacter spp, Serratia marcescens, Citrobacter freundii, Providencia spp and Morganella morganii, often referred to as the ESCPM group, may express high levels of chromosomal AmpC $\beta$-lactamases at high levels following exposure to $\beta$-lactams, mainly after third-generation cephalosporin therapy. ${ }^{1}$ The induction or selection of derepressed isolates is a concern because they contribute to the isolation of organisms no longer susceptible to specific $\beta$-lactams and may lead to clinical failure, with scarce antimicrobial options. ${ }^{2}$

Ceftolozane with tazobactam $(\mathrm{C} / \mathrm{T})$ is a combination drug comprising a $\beta$-lactamase inhibitor (tazobactam) with a new cephalosporin (ceftolozane). Tazobactam inhibits class A extendedspectrum $\beta$-lactamases (EBSLs), and ceftolozane acts via a high affinity for some penicillin-binding-protein (PBPs). C/T is stable in the presence of AmpC $\beta$-lactamases and against OprD deficiency and efflux pumps. These characteristics make the $\mathrm{C} / \mathrm{T}$ combination an important weapon in the treatment of infections due to extensively resistant $P$ seudomonas aeruginosa that are not carbapenemase producers. ${ }^{3}$

Despite the high efficacy described so far, emergence of resistance to $\mathrm{C} / \mathrm{T}$, mainly in $P$. aeruginosa isolates overexpressing AmpC- $\beta$-lactamase enzymes, have been reported. ${ }^{4}$ Although derepressed AmpC may occur in P. aeruginosa, the main target for $\mathrm{C} / \mathrm{T}$ use, this resistance mechanism is more robust in Enterobacter cloacae complex isolates, with a higher ability than others from

Author for correspondence: Leandro Reus Rodrigues Perez, E-mail: leandro.reus@ gmail.com

Cite this article: Perez LRR, Carniel E, and Narvaez GA. (2020). High minimum inhibitory concentrations among derepressed AmpC-beta-lactamase-producing Enterobacter cloacae complex isolates for ceftolozane with tazobactam. Infection Control \& Hospital Epidemiology, 41: 631-633, https://doi.org/10.1017/ice.2020.31 the ESCPM group to derepress AmpC- $\beta$-lactamase production, which has important clinical and therapeutic implications. ${ }^{5}$

The main objective of this study was to determine the $\mathrm{C} / \mathrm{T}$ minimum inhibitory concentration (MIC) among E. cloacae complex isolates, producing or not derepressed AmpC- $\beta$-lactamases. Additionally, meropenem and ceftazidime/avibactam MICs were also determined.

A set of 123 E. cloacae complex isolates recovered from inpatients between August 2016 and December 2017, in southern Brazil, were included in this study. Bacterial identification was made using the Vitek 2 automated system (bioMérieux, Marcy I'Etoile, France) and matrix-assisted laser desorption/ ionization time-of-flight mass spectroscopy (MALDI-TOF MS) for confirmation when necessary. Ertapenem susceptibility was determined using disc-diffusion testing. ${ }^{6}$ The MICs of cefotolozane/ tazobactam, meropenem, and ceftazidime/avibactam were determined using MIC test strips (MTS, Liofilchem, Italy) and were interpreted according to European Committee on Antimicrobial Susceptibility Testing (EUCAST) break points. ${ }^{6}$ To attribute the resistance mechanism for the selected E. cloacae complex isolates, a synergistic test was applied using an enzymatic inhibition testing with clavulanic acid and cloxacillin and/or phenyl-boronic acid to detect ESBLs and AmpC enzymes, in that order, as reported elsewhere. ${ }^{7}$ No isolate with carbapenemase production was included in this study, and for this study, all isolates were screened for a negative result using a blue-carba test to exclude class A and B carbapenemases and an OKN K-set immunochromatographic assay to exclude OXA-48-like production (ie, a carbapenemase with low hydrolysis activity for carbapenems and eventually resulting in a negative blue-carba test). ${ }^{8}$

Among the 123 isolates, 39 (31.7\%) and 84 (68.3\%) were characterized as derepressed and not-derepressed AmpC- $\beta$-lactamase 


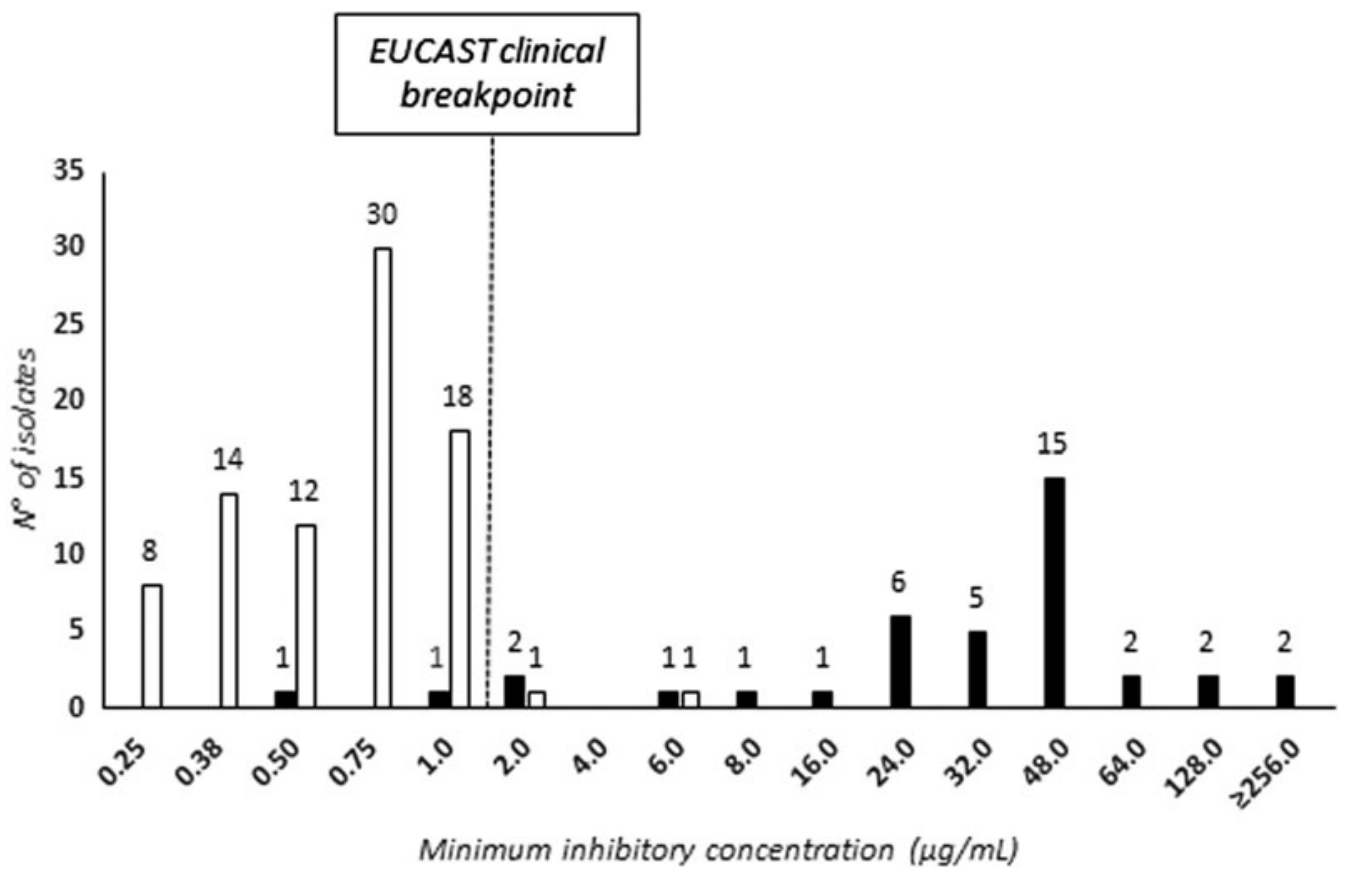

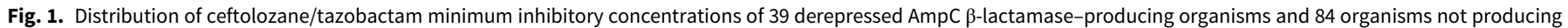

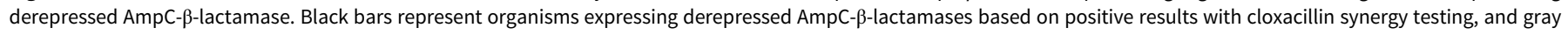
bars represent organisms not expressing derepressed AmpC- $\beta$-lactamases based on negative results with cloxacillin synergy testing.

producers, respectively, according to phenotypic testing. For derepressed AmpC- $\beta$-lactamase producers, 36 of 39 isolates (92.3\%) were resistant to ertapenem according to disc-diffusion testing. For all isolates, meropenem and ceftazidime/avibactam showed excellent activity: $\mathrm{MIC}_{90}$ of 0.75 and $2.0 \mathrm{mg} / \mathrm{L}$, respectively. No ceftazidime/avibactam resistance was observed among the isolates. However, 39 of 123 isolates (31.7\%) were resistant to $\mathrm{C} / \mathrm{T}$ and 37 of these 39 (94.9\%) were derepressed AmpC- $\beta$-lactamase producers. Only 2 derepressed AmpC isolates were susceptible to $\mathrm{C} / \mathrm{T}$ when EUCAST break points $(\leq 1.0$ and $>1.0 \mathrm{mg} / \mathrm{L}$, for susceptible and resistant, respectively) were considered (Fig. 1).

Enterobacter spp, particularly Enterobacter cloacae complex, are the most problematic pathogens because of their potential for derepressing AmpC $\beta$-lactamase production, presenting an approximate rate that is 10 -fold higher than that of other Enterobacterales. $^{9}$

Ceftolozane/tazobactam can overcome inactivation by ESBL $\beta$-lactamases, usually allowing maintenance of its activity against Enterobacterales producing the globally important ESBLs CTXM-14 and CTX-M-15. On the other hand, C/T may be influenced by AmpC $\beta$-lactamase activity, based on the results presented here, despite the fact that this drug combination is considered to have improved steric hindrance to prevent AmpC $\beta$-lactamasemediated hydrolysis.

High-level AmpC expression appears to confer a fitness cost to an organism because of the high metabolic energy required to express $a m p C$ regulation. Nevertheless, in the face of a persistent stimulus (eg, $\beta$-lactam exposure), this phenotype may be sustained, and for this reason, C/T (like many expanded-spectrum cephalosporins) may be discouraged for the treatment of infections caused by E. cloacae complex.

Some other points should also be considered. First, C/T was primarily designed to treat Pseudomonas infections, and because this bacterium is highly susceptible to this drug, ${ }^{10}$ it is reasonable to conclude that Pseudomonas usually does not derepress chromosomal AmpC enzymes, even those with an extensively drug-resistant phenotype. Second, C/T is an inappropriate drug for use in empirical therapeutic approaches; it should be preserved for the specific niche for Pseudomonas infection. Third, high inactivation of $\mathrm{C} / \mathrm{T}$ by derepressed $\mathrm{AmpC}$ organisms justifies strict monitoring of its resistance level in both Enterobacterales and Pseudomonas, particularly those associated with poor outcomes when high MICs $(>2 \mathrm{~g} / \mathrm{mL})$ are observed. ${ }^{10}$

In conclusion, in this study, we identified a mechanism that seems to be predictive of high $\mathrm{C} / \mathrm{T}$ resistance levels. More prominent among bacteria that are capable of derepressing AmpC $\beta$-lactamases, mainly the E. cloacae complex (but also Pseudomonas on a minor scale), this resistance to $\mathrm{C} / \mathrm{T}$ should lead to stricter use and monitoring of this drug.

Acknowledgments. The authors thank M.S.D. for providing the MIC test strips.

Financial support. No financial support was provided relevant to this article.

Conflicts of interest. All authors report no conflicts of interest relevant to this article.

\section{References}

1. Jacoby GA. AmpC beta-lactamases. Clin Microbiol Rev 2009;22:161-182.

2. Alvarez M, Tran JH, Chow N, Jacoby GA. Epidemiology of conjugative plasmid-mediated AmpC beta-lactamases in the United States. Antimicrob Agents Chemother 2004;48:533-537.

3. Craig WA, Andes DR. In vivo activities of ceftolozane, a new cephalosporin, with and without tazobactam against Pseudomonas aeruginosa and Enterobacteriaceae, including strains with extended-spectrum $\beta$-lactamases, in the thighs of neutropenic mice. Antimicrob Agents Chemother 2013;57:1577-1582.

4. Castanheira M, Mills JC, Farrell DJ, Jones RN. Mutation-driven beta-lactam resistance mechanisms among contemporary ceftazidime-nonsusceptible 
Pseudomonas aeruginosa isolates from US hospitals. Antimicrob Agents Chemother 2014;58:6844-6850.

5. Perez LRR. From dusk to dawn: understanding the impact of ertapenem resistance mechanisms on the in vitro potency of other drugs among Enterobacter cloacae complex isolates. Infect Control Hosp Epidemiol 2018;39:500-502.

6. Clinical breakpoints version 9.0. European Committee on Antimicrobial Susceptibility Testing (EUCAST) website. htpp://www.wucast.org/clinical_ breakpoints/. Updated January 1, 2019. Accessed November 12, 2019.

7. Perez, LR. Carbapenem-resistant Enterobacteriaceae: a major prevalence difference due to the high performance of carbapenemase producers when compared to the nonproducers. Infect Control Hosp Epidemiol 2015;36: 1480-1482.
8. Glupczynski Y, Jousset A, Evrard S, et al. Prospective evaluation of the OKN $\mathrm{K}-\mathrm{SeT}$ assay, a new multiplex immunochromatographic test for the rapid detection of OXA-48-like, KPC, and NDM carbapenemases. J Antimicrob Chemother 2017;72:1955-1960.

9. Pfaller MA, Jones RN, Marshall SA, et al. Inducible AmpC beta-lactamaseproducing gram-negative bacilli from blood stream infections: frequency, antimicrobial susceptibility, and molecular epidemiology in a national surveillance program (SCOPE). Diagn Microbiol Infect Dis 1997;28:211-219.

10. Rodríguez-Núñez O, Periañez-Parraga L, Oliver A, et al. Higher MICs $(>2 \mathrm{mg} / \mathrm{L})$ predict 30 -day mortality in patients with lower respiratory tract infections caused by multidrug- and extensively drug-resistant Pseudomonas aeruginosa treated with ceftolozane/tazobactam. Open Forum Infect Dis 2019;6:ofz416. 